Supporting Information:

\title{
Weakly Conjugated Phosphine Oxide Hosts for Efficient Blue Thermally Activated Delayed Fluorescence OLEDs
}

Ke Duan, $\ddagger^{a}$ Dan Wang, $\ddagger^{a}$ Ming Yang, ${ }^{a}$ Ziyang Liu, ${ }^{b}$ Chao Wang, ${ }^{c}$ Taiju Tsuboi, ${ }^{a}$ Chao Deng, ${ }^{a}$ Qisheng Zhang* ${ }^{a}$

a MOE Key Laboratory of Macromolecular Synthesis and Functionalization, Department of Polymer Science and Engineering, Zhejiang University, Hangzhou 310027, China.

${ }^{b}$ Key Lab of Organic Optoelectronics and Molecular Engineering of Ministry of Education, Department of Chemistry and Center for Flexible Electronics Technology, Tsinghua University, Beijing 100084, China.

c Singapore University of Technology and Design, 8 Somapah Road, 487372, Singapore.

* E-mail: qishengzhang@zju.edu.cn

$\$$ These authors contributed equally. 


\section{Contents}

1. Supplemental Figures

2. Experimental Section

2.1 Materials and measurements

2.2 Synthesis

2.3 Photoluminescence measurements

2.4 Electrochemical measurements

2.5 Quantum chemical calculations

2.6 Device fabrication and measurements

3. References 


\section{Supplemental Figures}

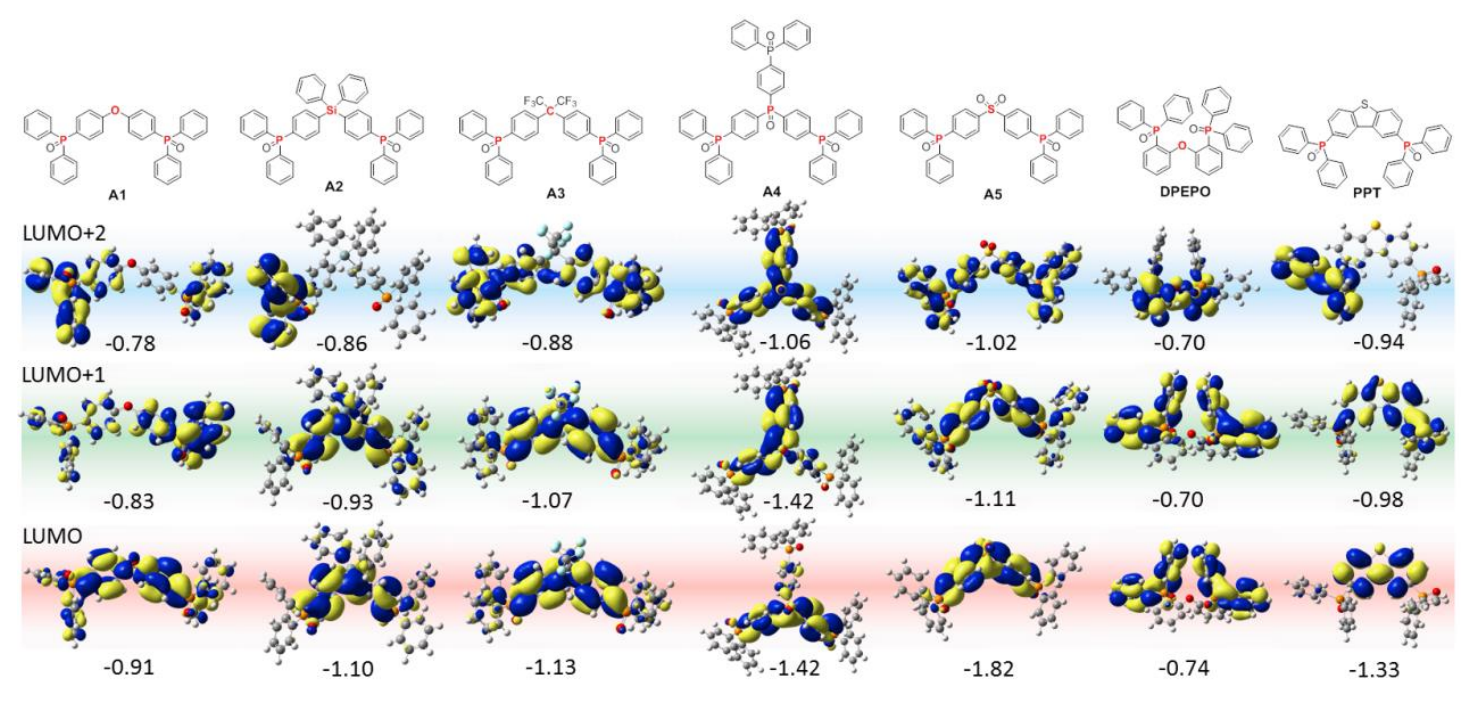

Figure S1. Distribution and energies of LUMO, LUMO+1 and LUMO+2 for A1-A5, DPEPO and PPT in their $\mathrm{S}_{0}$ state in vacuum. The geometries are optimized at the DFT/B3LYP/6-31G* level.

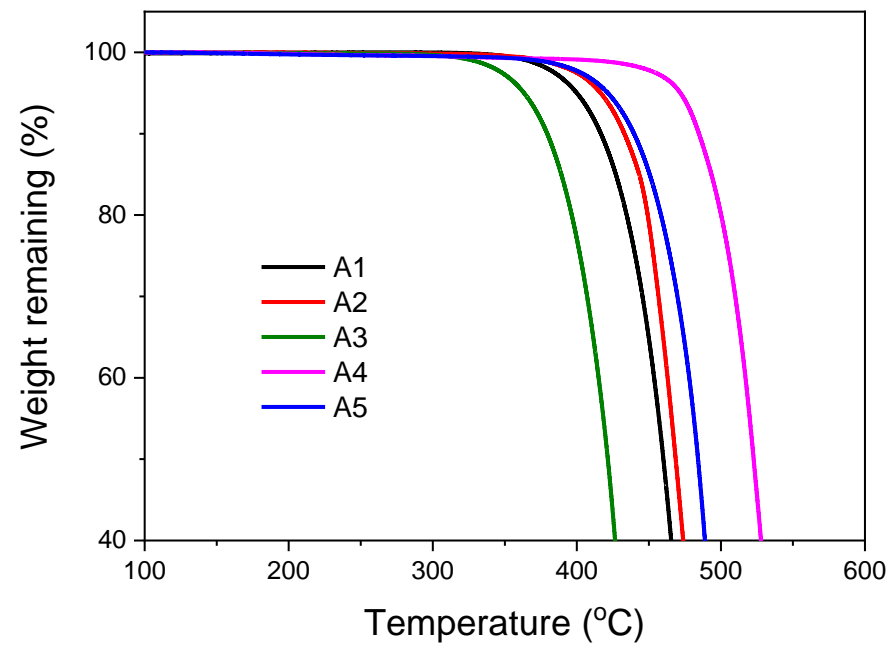

Figure S2. Thermogravimetry analysis (TGA) curves of A1-A5 under nitrogen flow. The $5 \mathrm{wt} \%$ weight loss temperatures for $\mathbf{A 1}-\mathbf{A 5}$ are determined to be $400^{\circ} \mathrm{C}, 417^{\circ} \mathrm{C}$, $365^{\circ} \mathrm{C}, 472^{\circ} \mathrm{C}$, and $421^{\circ} \mathrm{C}$, respectively. 


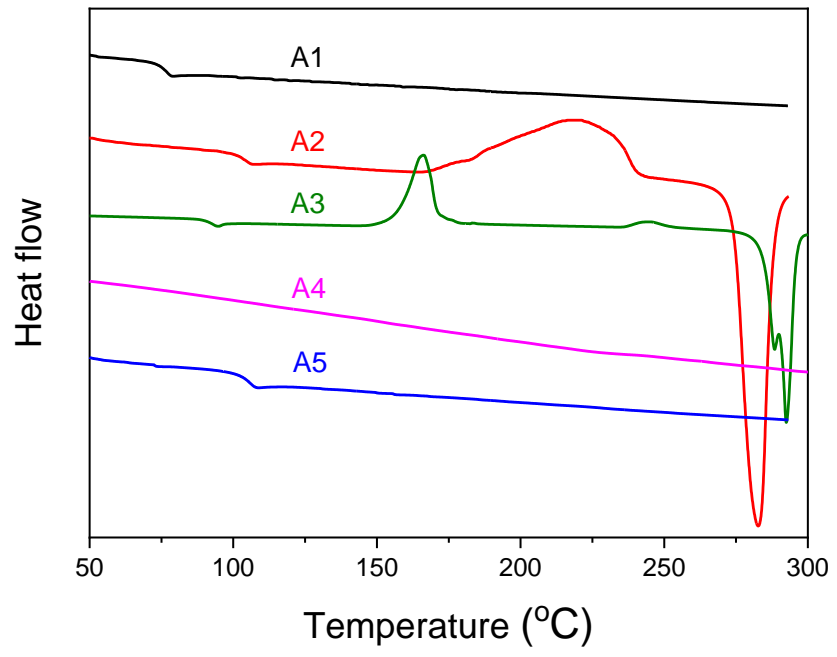

Figure S3. Differential scanning calorimetry (DSC) curve of $\mathbf{A 1}-\mathbf{A 5}$ under nitrogen flow. The glass transition temperatures $\left(T_{\mathrm{g}}\right)$ of $\mathbf{A 1}, \mathbf{A 2}, \mathbf{A 3}, \mathbf{A 5}$ are estimated to be $80^{\circ} \mathrm{C}, 103^{\circ} \mathrm{C}, 92^{\circ} \mathrm{C}$ and $106^{\circ} \mathrm{C}$, respectively.

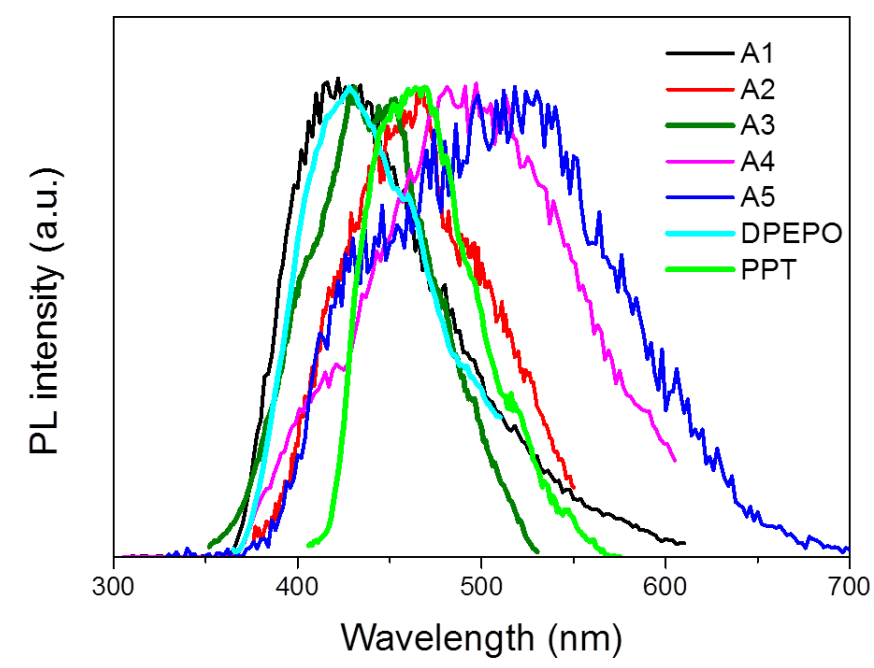

Figure S4. Phosphorescence spectra (0.1-1 ms) of the neat films of A1-A5, DPEPO and PPT at $77 \mathrm{~K}$. 

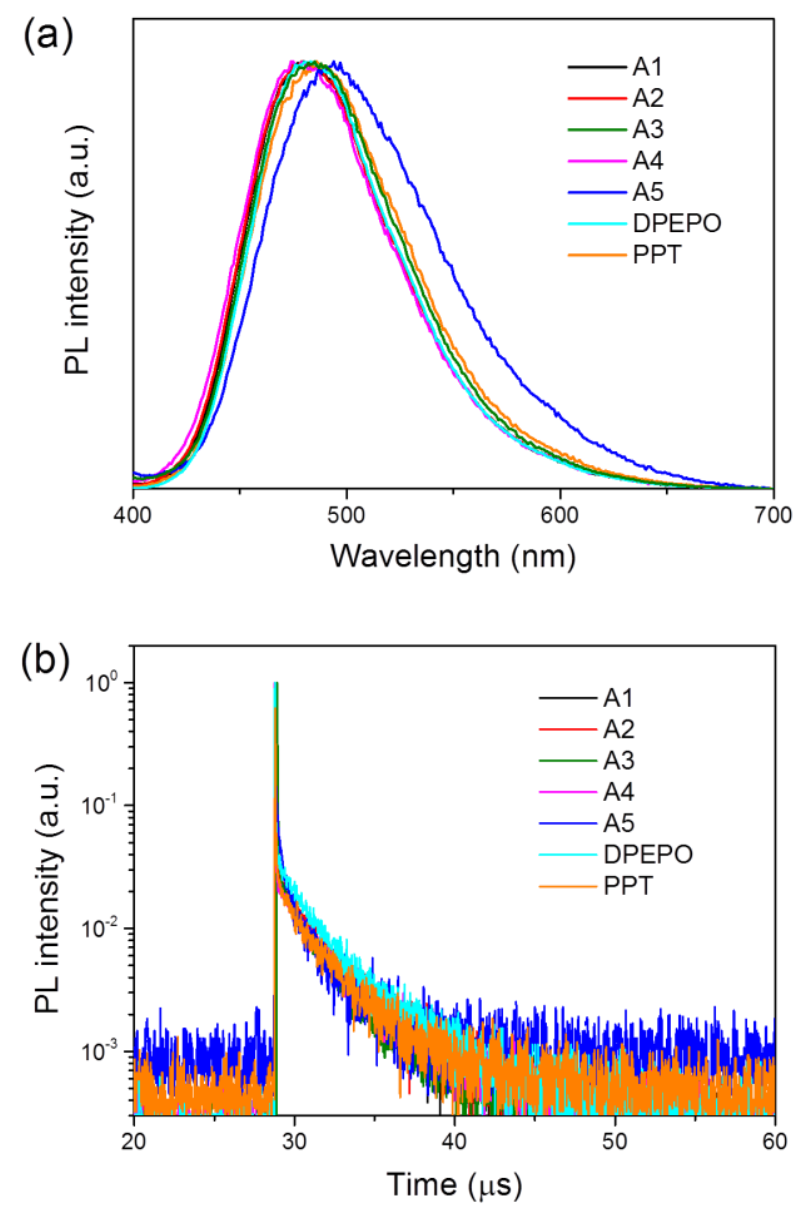

Figure S5. Steady state (a) and transient decay (b) spectra of DMAC-DPS doped into the films of A1-A5, DPEPO and PPT (10 wt\%) at room temperature. The PLQYs of these doped films were measured to be 0.89 for $\mathbf{A 1}, 0.85$ for $\mathbf{A 2}, 0.90$ for $\mathbf{A 3}, 0.77$ for A4, 0.34 for A5, 0.90 for DPEPO, and 0.72 for PPT. 


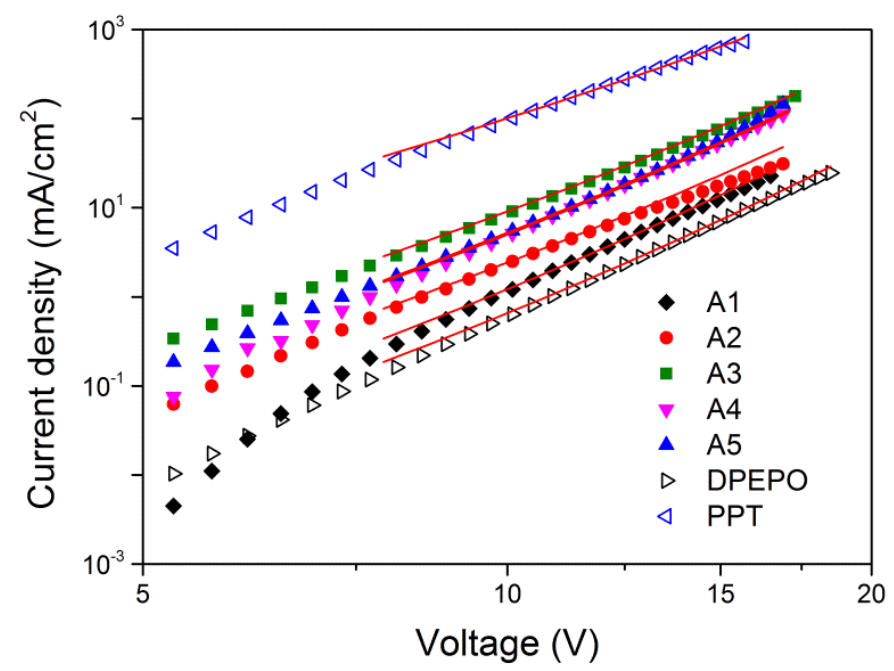

Figure S6. Current density-voltage characteristics of the electron-only devices and the fitting curves according to a modified SCLC model. The current density $J$ is given by $J=(9 / 8) \varepsilon \varepsilon_{0} \mu_{0} \exp \left[0.89 \beta(V / d)^{1 / 2}\right] V^{2} / d^{3}$, where $\varepsilon, \varepsilon_{0}, \mu_{0}, V$ and $d$ are the dielectric constant, the permittivity of free space, the mobility of free space, the applied voltage and the thickness of the film, respectively. ${ }^{1}$ In this calculation, $\varepsilon=3$ was employed. ${ }^{1}$ 

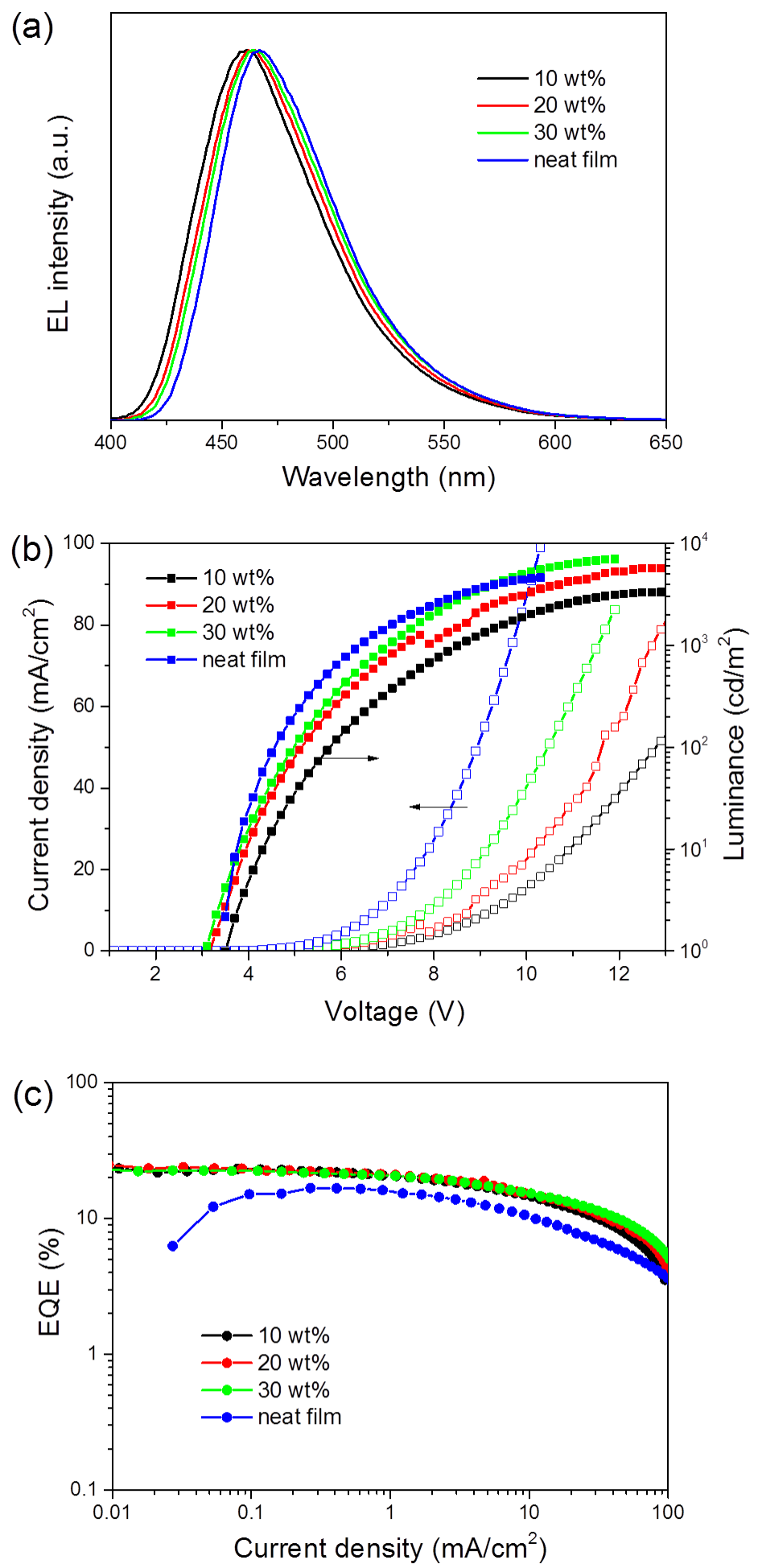

Figure S7. (a) EL spectra at $10 \mathrm{~mA} / \mathrm{cm}^{2}$, (b) luminance-current density-voltage characteristics, (c) EQE-current density characteristics of DMAC-DPS-based OLEDs with $\mathbf{A 3}$ host and different doping concentrations. Device structure: ITO/ $\mathrm{MoO}_{3}(3 \mathrm{~nm})$ /mCP (40 nm)/x wt\% DMAC-DPS: A3 (30 nm)/A3 (40 nm)/Liq (2 nm)/Al (120 nm) 

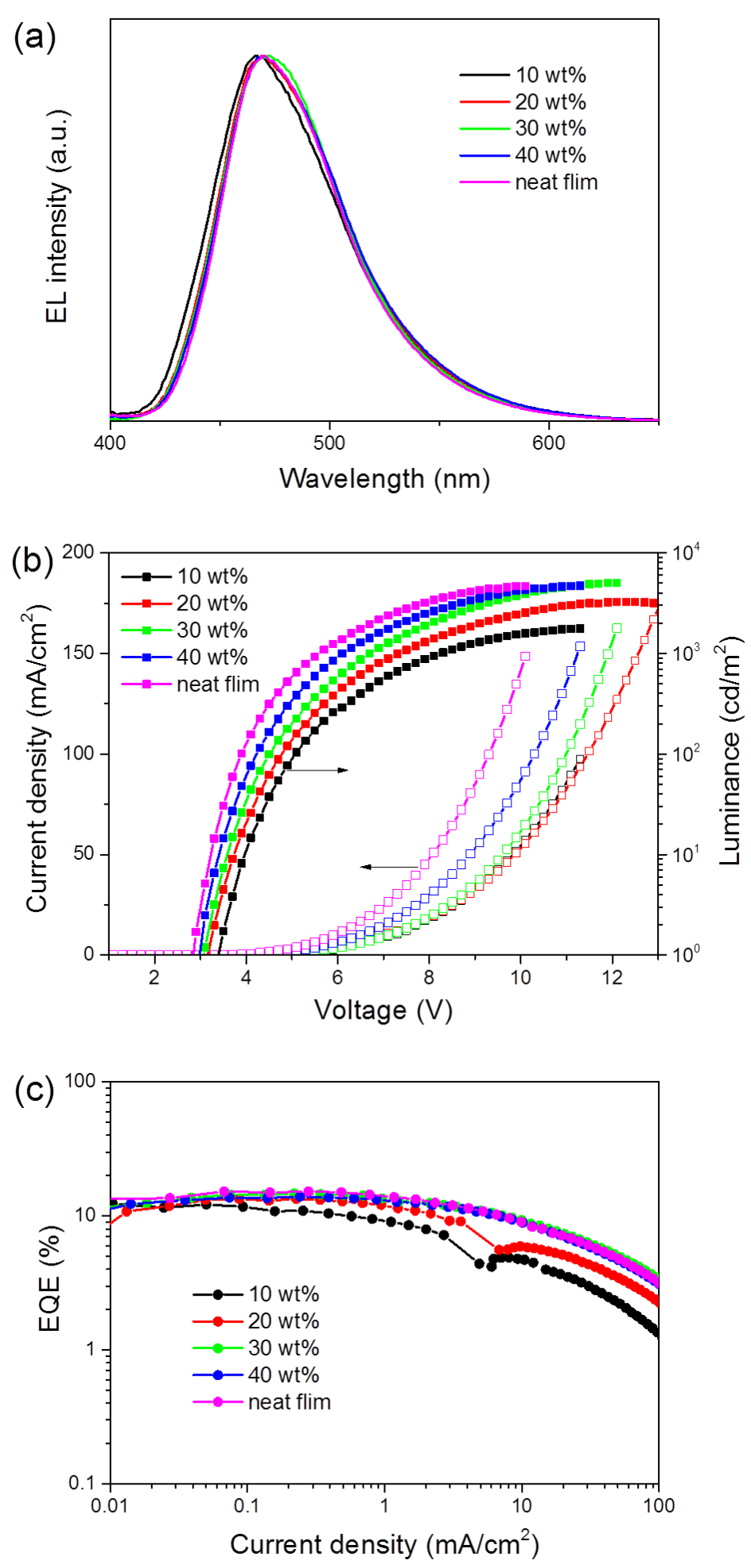

Figure S8. (a) EL spectra at $10 \mathrm{~mA} / \mathrm{cm}^{2}$, (b) luminance-current density-voltage characteristics, (c) EQE-current density characteristics of DMAC-DPS-based OLEDs with A4 host and different doping concentrations. Device structure: ITO/ $\mathrm{MoO}_{3}(3 \mathrm{~nm})$ /mCP (40 nm)/x wt\% DMAC-DPS: A4 (30 nm)/PPT (40 nm)/Liq (2 nm)/Al (120 nm) 

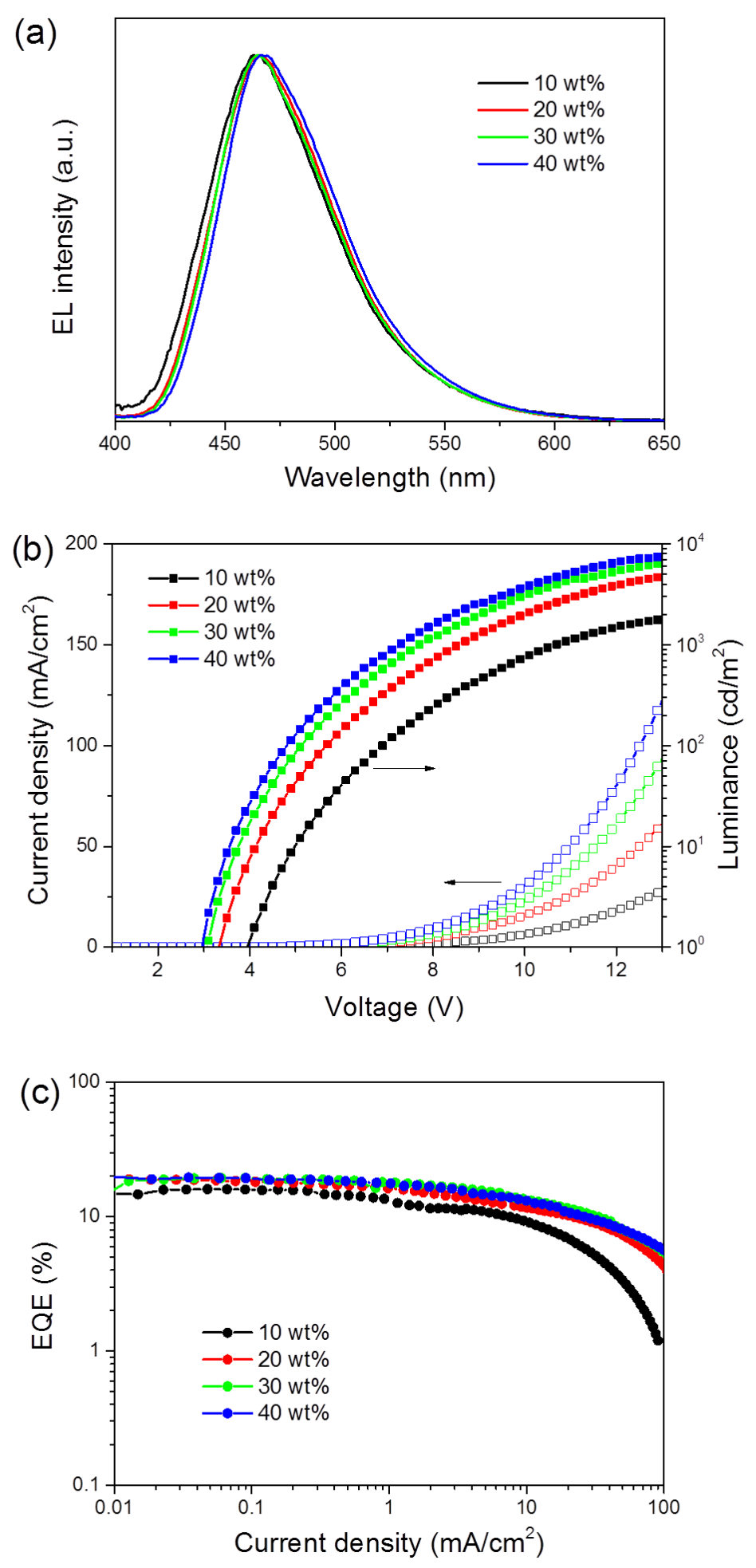

Figure S9. (a) EL spectra at $10 \mathrm{~mA} / \mathrm{cm}^{2}$, (b) luminance-current density-voltage characteristics, (c) EQE-current density characteristics of DMAC-DPS-based OLEDs with DPEPO host and different doping concentrations. Device structure: ITO/ $\mathrm{MoO}_{3}(3$ $\mathrm{nm}) / \mathrm{mCP}(40 \mathrm{~nm}) / \mathrm{x}$ wt\% DMAC-DPS: DPEPO $(30 \mathrm{~nm}) / \mathrm{PPT}(40 \mathrm{~nm}) / \mathrm{Liq}(2 \mathrm{~nm}) / \mathrm{Al}$ $(120 \mathrm{~nm})$ 


\section{Experimental Section}

\subsection{Materials and measurement:}

All solvents and starting materials were purchased from commercial resources and were used as received unless otherwise stated. DPEPO and A1 were prepared by the procedures in the literature, ${ }^{2,3}$ and were further purified by sublimation twice. The intermediates 2,2-bis(4-aminophenyl)hexafluoropropane, chlorodiphenylphosphine, tris(4-fluorophenyl)phosphine and triphenylphosphine were purchased from Bidepharm Co., Ltd. and were used without further purification. Other OLED materials $\mathrm{MoO}_{3}, \mathrm{mCP}, \mathrm{PPT}$ and Liq were purchased from Jilin Optical and Electronic Materials Co., Ltd. and were used without further purification.

Nuclear magnetic resonance spectroscopy (NMR) was recorded on a Bruker Avance III 400 spectrometer $\left({ }^{1} \mathrm{H}: 400 \mathrm{MHz}\right.$ and $\left.{ }^{13} \mathrm{C}: 100 \mathrm{MHz}\right)$ at room temperature using $\mathrm{CDCl}_{3}$ as solvent and tetramethylsilane (TMS) as internal reference. Mass spectra measurements were performed on a IT-TOF (Shimadzu, Japan) in positive ion mode ESI-MS. Accurate mass determination was corrected by calibration using sodium trifluoroacetate clusters as reference.

\subsection{Synthesis:}

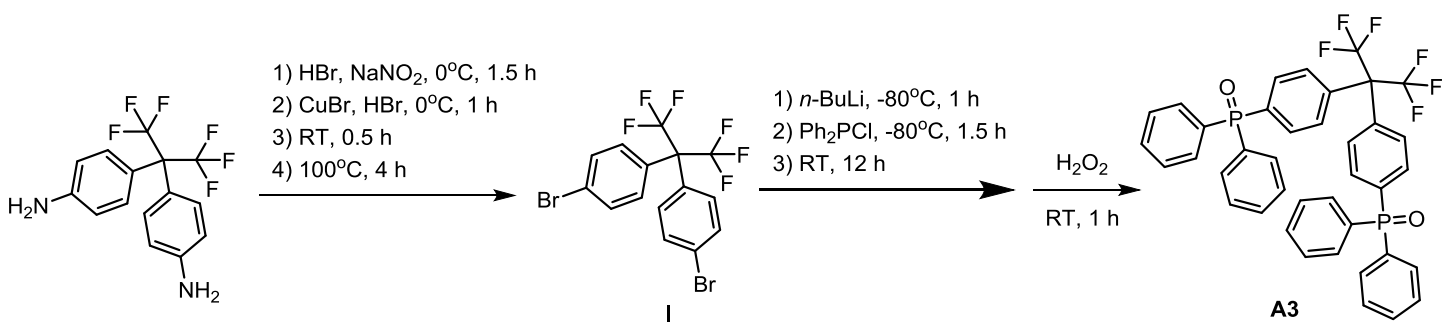

Scheme S1. Synthesis toward A3.

4,4'-(Perfluoropropane-2,2-diyl)bis(bromobenzene) (I): 2,2-Bis(4-aminophenyl)hexafluoropropane $(1.337 \mathrm{~g}, 4 \mathrm{mmol})$ and $\mathrm{HBr}(5 \mathrm{ml}, 28 \mathrm{mmol})$ were added to a $50 \mathrm{ml}$ single-mouth flask and dissolved in $8 \mathrm{ml}$ water by ultrasound. $4 \mathrm{ml} \mathrm{NaNO}_{2}$ aqueous solution $(0.84 \mathrm{~g}, 8.4 \mathrm{mmol})$ was slowly added to the mixture cooled in an ice-water bath. After stirring for 1.5 hours at $0-5^{\circ} \mathrm{C}$, earthy yellow diazonium salt was formed. 
$\mathrm{CuBr}(1.72 \mathrm{~g}, 8.4 \mathrm{mmol})$ and $\mathrm{HBr}(9 \mathrm{ml}, 56 \mathrm{mmol})$ were added to a $250 \mathrm{ml}$ single-mouth flask and then stirred at $0-5^{\circ} \mathrm{C}$. The diazonium salt was added into the flask, generating a large amount of gas. The reaction continued for 1 hour at $0-5^{\circ} \mathrm{C}$, then for 0.5 hour at room temperature (RT) and 4 hours at $100^{\circ} \mathrm{C}$. The reddish black liquid was extracted with dichloromethane (DCM) and was subjected to silica gel column chromatography using petroleum ether (PE) as eluent to afford a solid (I) with a yield of $60 \%$.

\section{1,1'-\{[2,2,2-Trifluoro-1-(trifluorome`thyl)ethylidene]di-4,1-phenylene $\}$ bis[1,1-}

diphenylphosphine oxide] (A3): I (1.109 g, $2.4 \mathrm{mmol})$ and $30 \mathrm{ml}$ dry tetrahydrofuran (THF) were added to a $250 \mathrm{ml}$ single-mouth long-neck reaction flask under the protection of nitrogen and stirred at $-80^{\circ} \mathrm{C}$. $2.5 \mathrm{M}$ butyl lithium $(2.1 \mathrm{ml}, 5.16 \mathrm{mmol})$ was added to react for 1 hour at $-80^{\circ} \mathrm{C}$. Chlorodiphenylphosphine $(1.165 \mathrm{~g}, 0.95 \mathrm{ml}$, $5.28 \mathrm{mmol}$ ) was add to react for 1.5 hours at $-80^{\circ} \mathrm{C}$ and then overnight at RT. Water was added into the solution and stirred for $10 \mathrm{~min}$. The mixture was extracted with DCM, concentrated and purified by silica gel column chromatography (DCM: PE $=1$ : 20) to obtain a white solid of $1.15 \mathrm{~g}$. The intermediate was dissolved in $20 \mathrm{ml}$ DCM. Hydrogen peroxide $(50 \mathrm{mmol})$ was slowly added to the solution and then stired for 1 hour at RT. After extraction and concentration, a white powder (A3) of $1.0 \mathrm{~g}$ was obtained with a total yield of $60 \% .{ }^{1} \mathrm{H} \mathrm{NMR}\left(\mathrm{CDCl}_{3}, 400 \mathrm{MHz}\right): \delta[\mathrm{ppm}] 7.74-7.64$ $(\mathrm{m}, 12 \mathrm{H}), 7.62-7.58(\mathrm{~m}, 4 \mathrm{H}), 7.52-7.47(\mathrm{~m}, 12 \mathrm{H}) .{ }^{13} \mathrm{C} \mathrm{NMR}\left(\mathrm{CDCl}_{3}, 100 \mathrm{MHz}\right): \delta$ [ppm] 136.7, 134.8, 133.8, 132.3, 132.3, 132.1, 132.0, 131.0, 130.3, 130.2, 128.8, 128.6, 77.3, 77.0, 76.7. HRMS $m / z: 704.24[\mathrm{M}]^{+}$. 
<smiles>Fc1ccc(P(c2ccc(F)cc2)c2ccc(F)cc2)cc1</smiles>

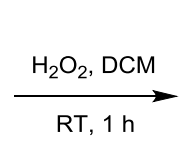

$\mathrm{RT}, 1 \mathrm{~h}$<smiles>O=P(c1ccc(F)cc1)(c1ccc(F)cc1)c1ccc(F)cc1</smiles>

II

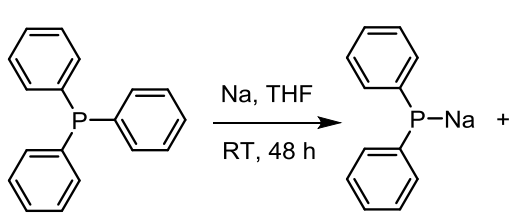<smiles>O=P(c1ccc(F)cc1)(c1ccc(F)cc1)c1ccc(F)cc1</smiles>
$\stackrel{\text { 1) }-40^{\circ} \mathrm{C}, 1 \mathrm{~h}}{\text { 2) } \mathrm{RT}, 2 \mathrm{Oh}} \underset{\mathrm{RT}, 1 \mathrm{~h}}{\longrightarrow} \frac{\mathrm{H}_{2} \mathrm{O}_{2}}{\longrightarrow}$<smiles>O=P(c1ccccc1)(c1ccccc1)c1ccc(P(=O)(c2ccccc2)c2ccccc2)cc1</smiles>

Scheme S2. Synthesis toward A4.

Tris(4-fluorophenyl)phosphine oxide (II) was obtained from tris(4-fluorophenyl) phosphine by a method same as that for $\mathbf{A 3}$.

Tris[4-(diphenylphosphinyl)phenyl]phosphine oxide (A4): Triphenylphosphine (30 mmol, $7.87 \mathrm{~g})$, dry THF (90 ml) and sodium (2.8 g, $120 \mathrm{mmol})$ were added to a three-necked flask under nitrogen protection and reacted at RT for 2 days. The excess sodium was removed from the dark red solution under nitrogen. The solution was cooled to $-40^{\circ} \mathrm{C}$ and II $(10 \mathrm{mmol})$ in $15 \mathrm{ml}$ THF was slowly added. The mixture was reacted for 1 hour at $-40^{\circ} \mathrm{C}$, and then for 20 hours at RT. After the removement of the solvent, the solid was redisolved in $100 \mathrm{ml}$ DCM. Hydrogen peroxide (100 mmol) was slowly added to the DCM solution cooled in an ice-water bath, and then reacted for 1 hour at RT. The organic layer was separated, washed by water and dried by anhydrous sodium sulfite. The crude product was purified by silica gel column chromatography using 20:1 DCM/methanol as eluent, giving the desired compound as a white powder $(4.5 \mathrm{~g}, 50 \%) .{ }^{1} \mathrm{H} \mathrm{NMR}\left(\mathrm{CDCl}_{3}, 400 \mathrm{MHz}\right): \delta[\mathrm{ppm}] 7.81-7.71(\mathrm{~m}$,

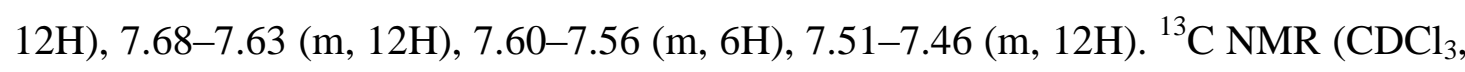
$100 \mathrm{MHz}): \delta[\mathrm{ppm}] 138.4,137,4,135.6,135.6,134.6,132.4,132.4,132.4,132.3$, 132.2, 132.1, 132.1, 132.0,132.0, 131.9, 131.9, 131.8, 130.8, 128.8, 128.7, 77.4, 77.1, 76.7. HRMS $m / z: 878.24[\mathrm{M}]^{+}$. 


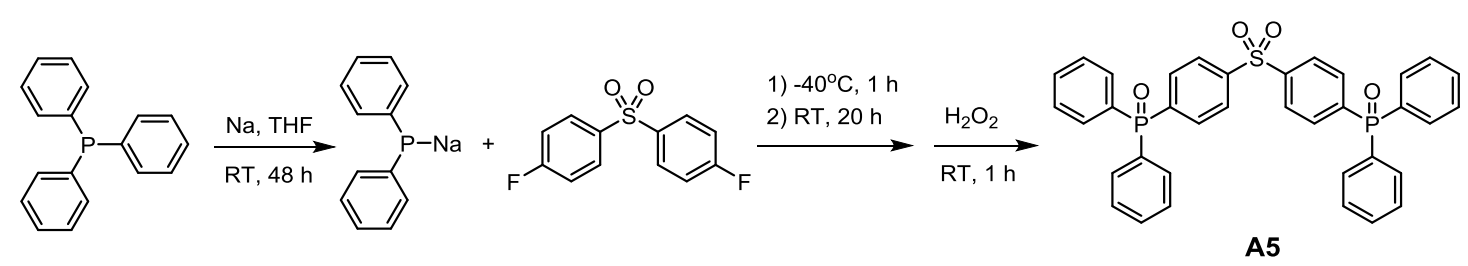

Scheme S3. Synthesis toward A5.

\section{1,1'-(sulfonyldi-4,1-phenylene)bis(1,1-diphenylphosphine oxide) (A5):}

Triphenylphosphine (50 mmol, $13.1 \mathrm{~g})$, dry THF $(150 \mathrm{ml})$ and sodium $(2.3 \mathrm{~g} 100$ mmol) were added to a three-necked flask under nitrogen protection and reacted at RT for 2 days. The excess sodium was removed from the dark red solution under nitrogen. The solution was cooled to $-40^{\circ} \mathrm{C}$ and bis( $p$-fiuorophenyl)sulfone $(6.1 \mathrm{~g}, 24 \mathrm{mmol})$ in $30 \mathrm{ml}$ THF was slowly added. The mixture was reacted for 1 hour at $-40^{\circ} \mathrm{C}$, and then for 20 hours at RT. After the removement of the solvent, the solid was redisolved in $200 \mathrm{ml}$ DCM. Hydrogen peroxide $(200 \mathrm{mmol})$ was slowly added to the DCM solution cooled in an ice-water bath, and then reacted for 1 hour at RT. The organic layer was separated, washed by water and dried by anhydrous sodium sulfite. The crude product was recrystallized from methanol and diethyl ether to obtain a colorless crystal of 8.0 $\mathrm{g}(54 \%$ yield $) .{ }^{1} \mathrm{H} \mathrm{NMR}\left(\mathrm{CDCl}_{3}, 400 \mathrm{MHz}\right): \delta[\mathrm{ppm}] 8.01(\mathrm{dd}, J=8.5,2.2 \mathrm{~Hz}, 4 \mathrm{H})$, 7.85-7.80 (m, 4H), 7.66-7.60 (m, 8H), 7.59-7.56 (m, 4H), 7.51-7.47 (m, 8H). ${ }^{13} \mathrm{C}$ NMR $\left(\mathrm{CDCl}_{3}, 100 \mathrm{MHz}\right): \delta[\mathrm{ppm}] 143.9,143.9,139.8,138.9,133.2,133.1,132.6$, 132.5, 132.0, 132.0, 131.9, 131.5, 130.5, 128.9, 128.8, 127.8, 127.7, 77.4, 77.0, 76.7. HRMS $m / z: 618.11[\mathrm{M}]^{+}$. 


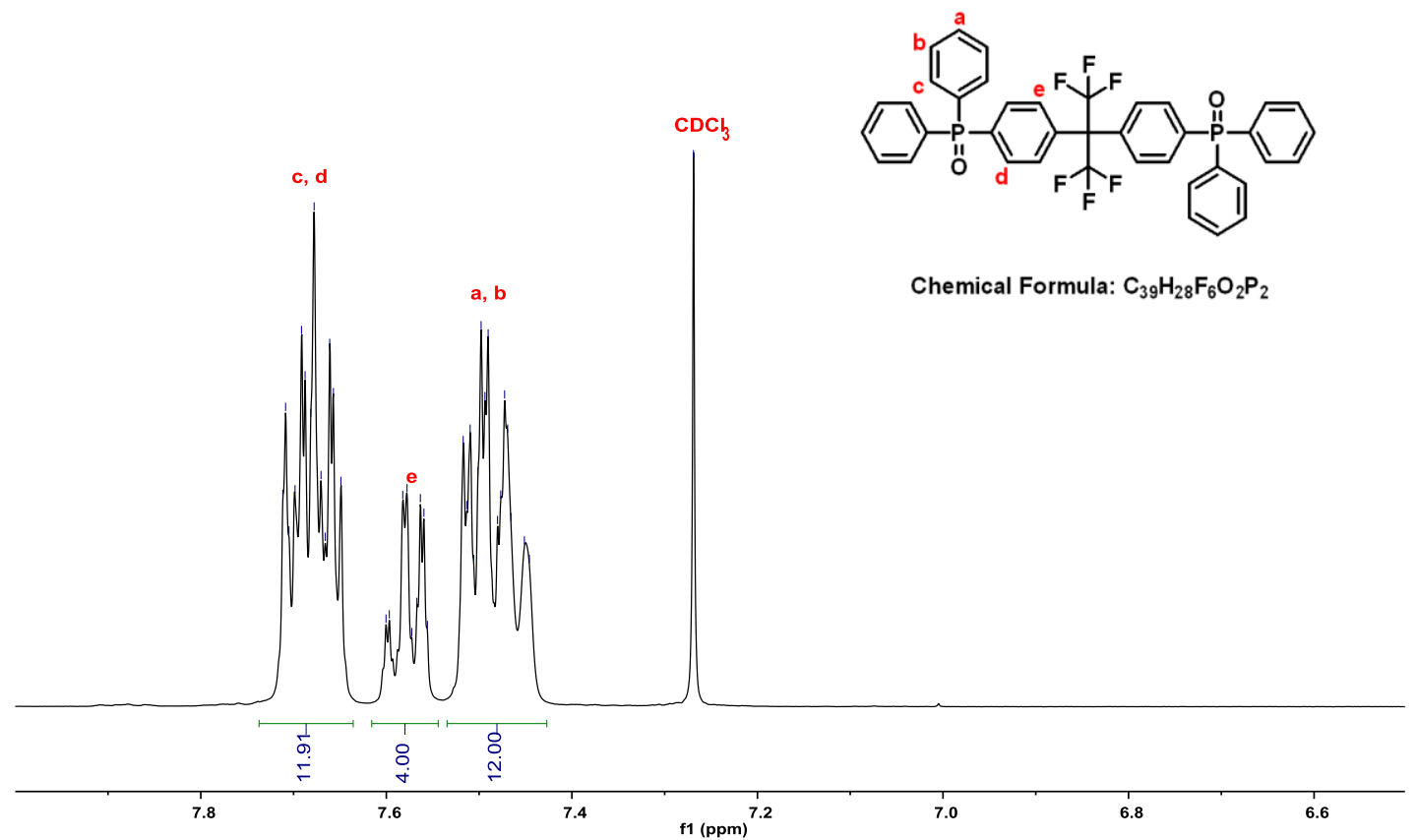

Figure S10. ${ }^{1} \mathrm{H}$ NMR spectra of A3.

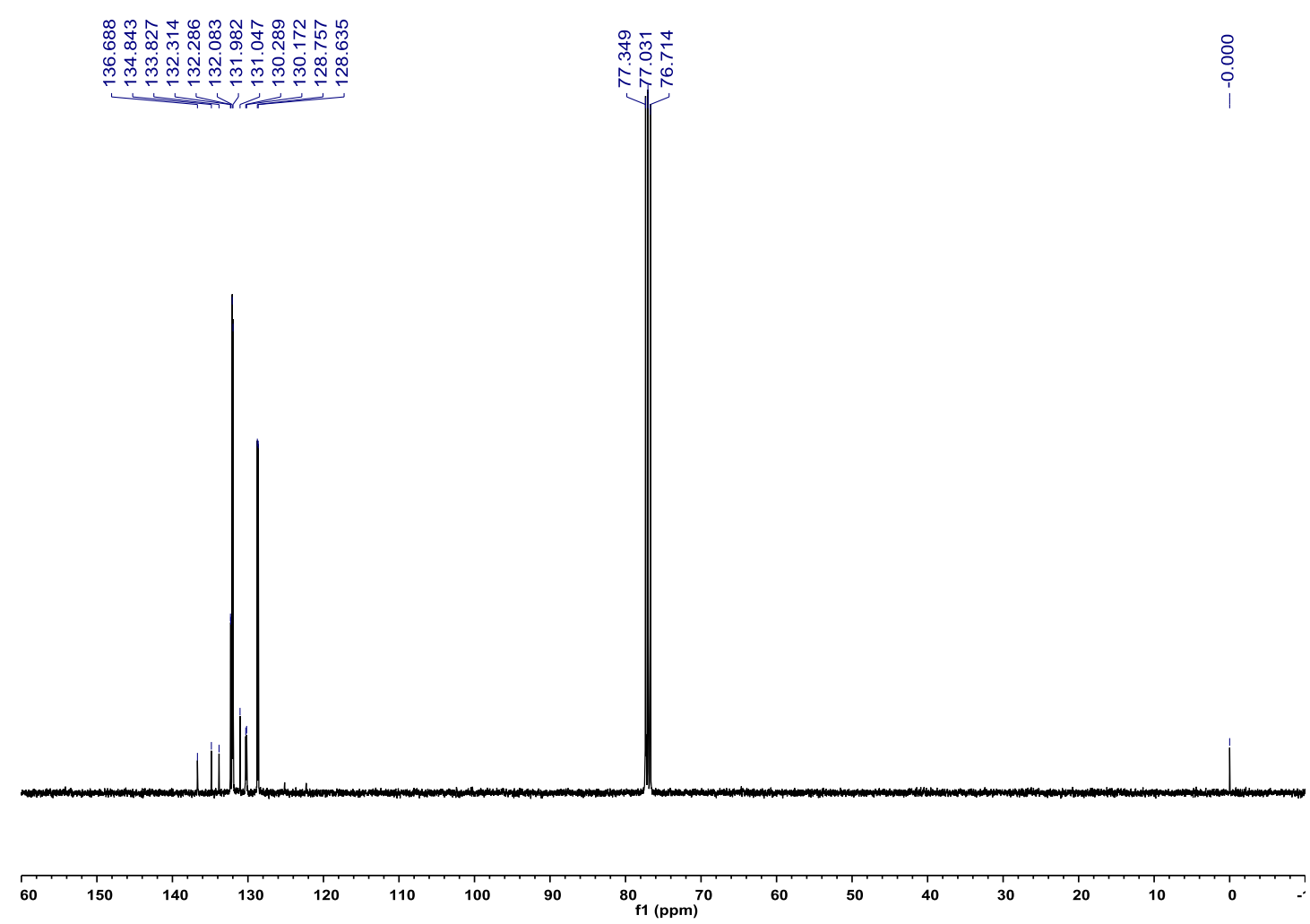

Figure S11. ${ }^{13} \mathrm{C}$ NMR spectra of A3. 


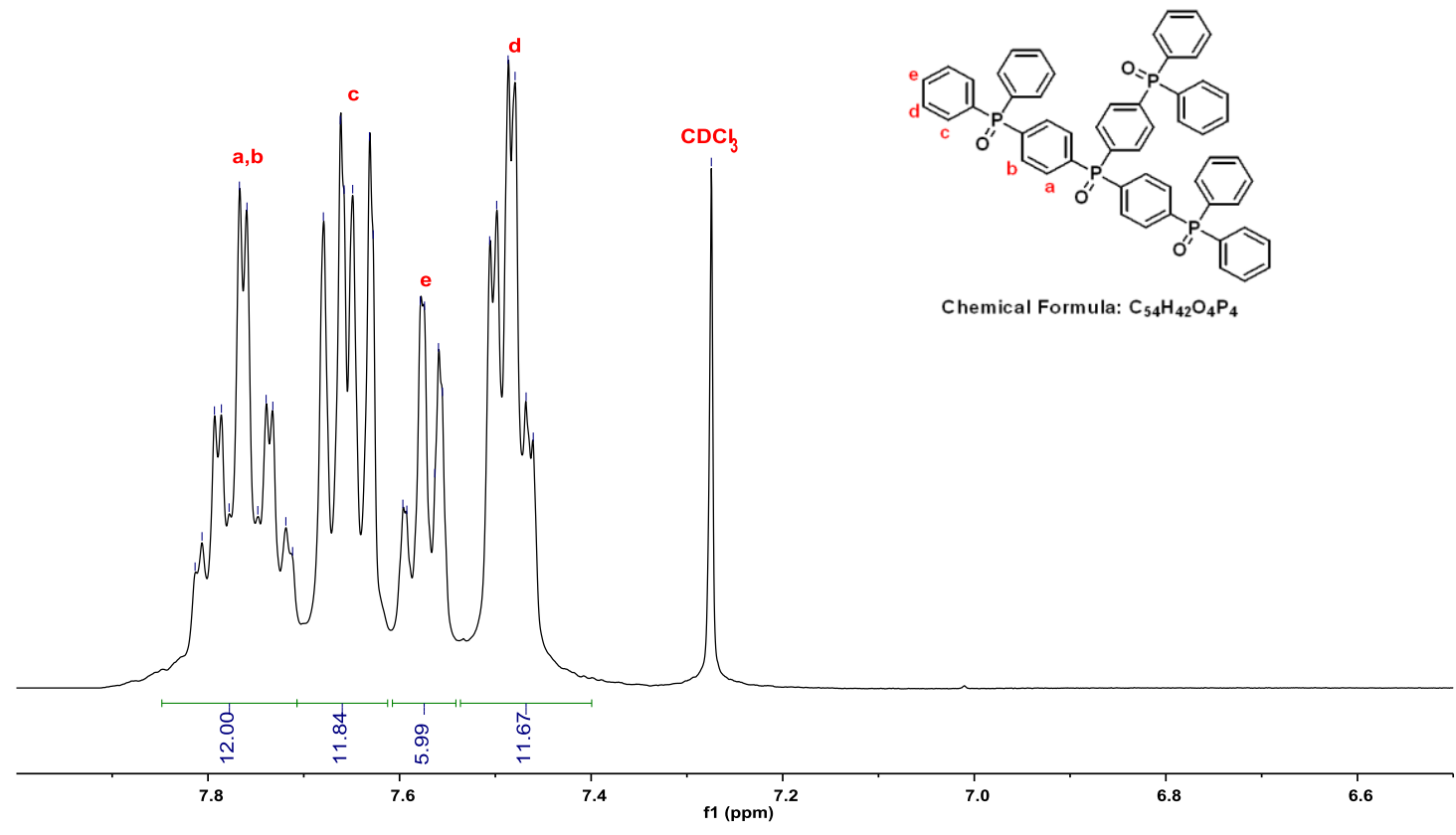

Figure S12. ${ }^{1}$ H NMR spectra of A4.

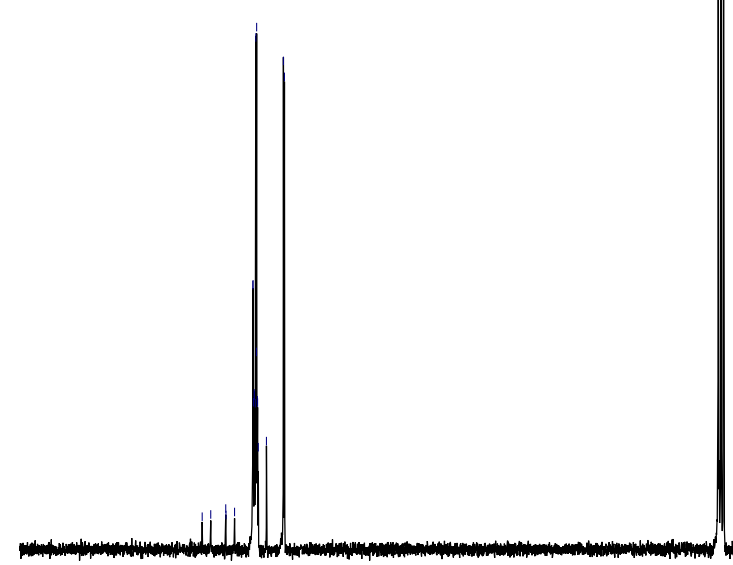

Figure S13. ${ }^{13} \mathrm{C}$ NMR spectra of A4. 


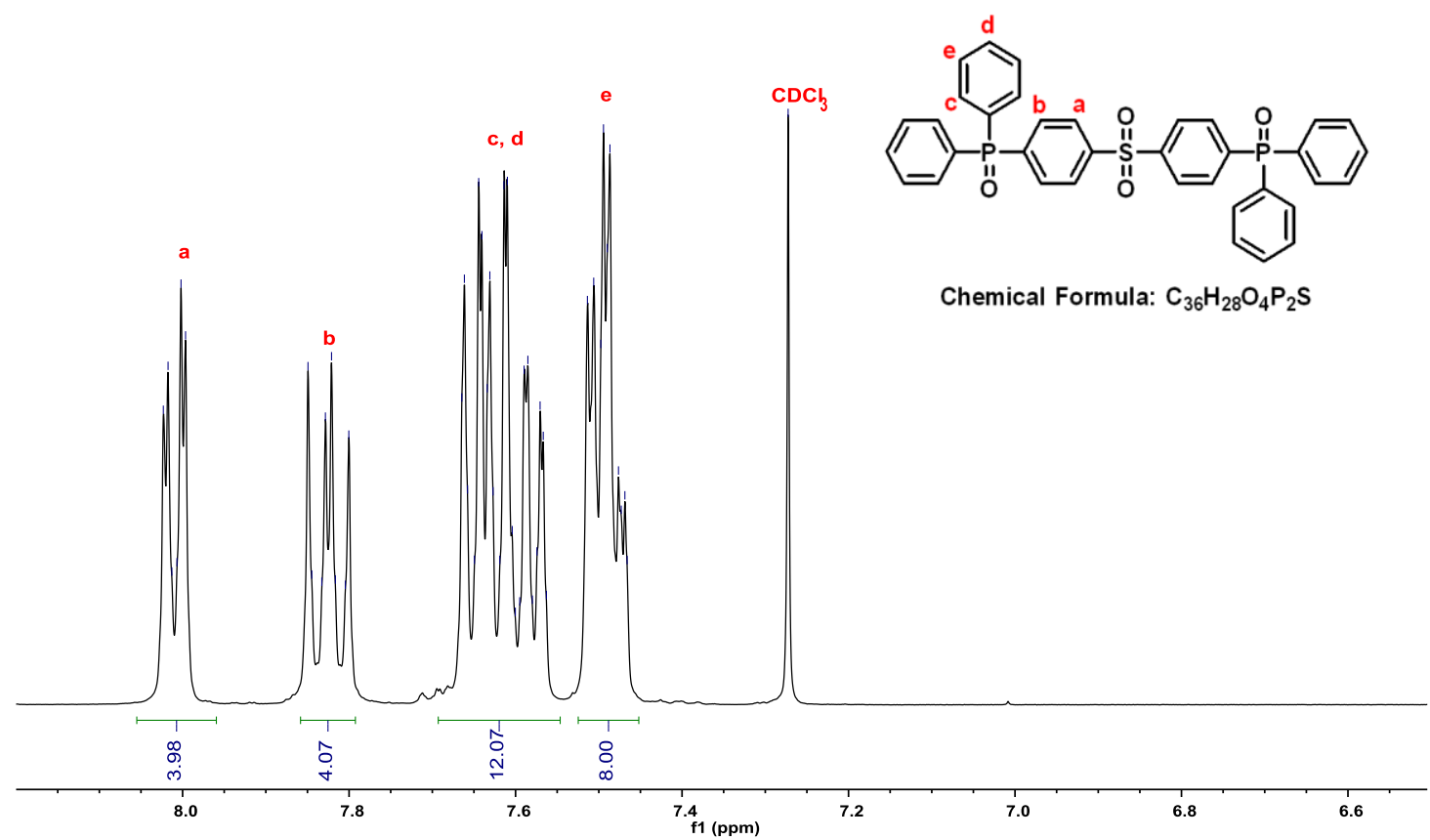

Figure S14. ${ }^{1} \mathrm{H}$ NMR spectra of A5.
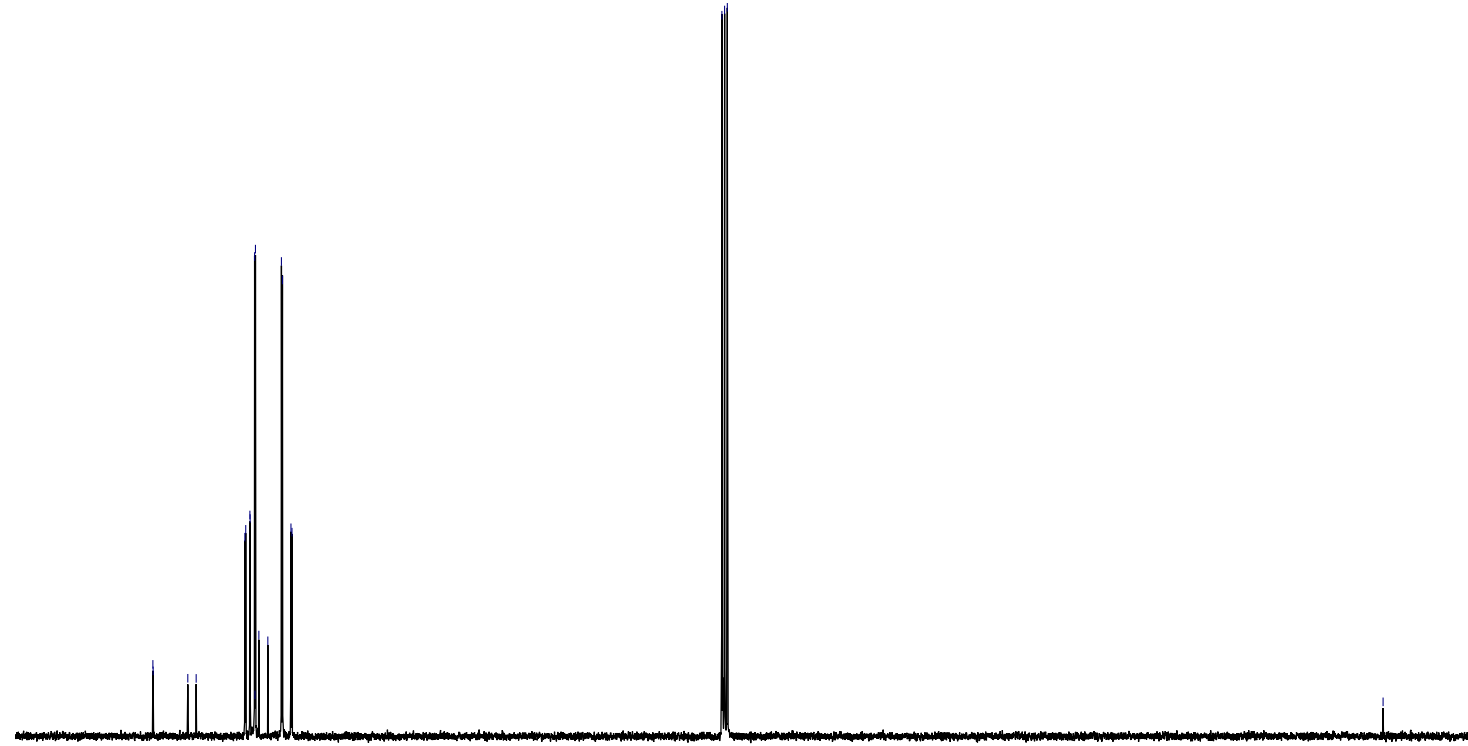

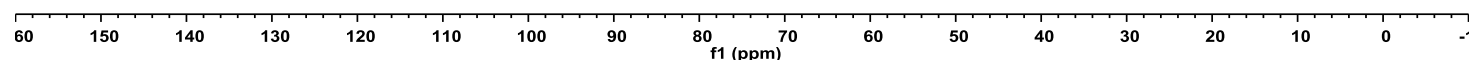

Figure S15. ${ }^{13} \mathrm{C}$ NMR spectra of A5. 


\subsection{Photoluminescence measurements:}

The absorption and emission spectra were recorded using a Shimadzu UV-2600 spectrophotometer and a PTI QM-40 spectrofluorometer, respectively. The photoluminescence quantum yields (PLQYs) of the film samples were measured using the PTI spectrofluorometer equipped with an integrating sphere. The low-temperature phosphorescence $(0.1-1 \mathrm{~ms})$ spectra were collected by the PTI spectrofluorometer equipped with an electric shutter and a PTI nitrogen laser (GL-3300, $\lambda=266 \mathrm{~nm}$, pulse width $\sim 1 \mathrm{~ns}$, pulse energy=1.45 mJ). The phosphorescence spectra of solutions were measured in a PTI WG-850-Q quartz Dewar with liquid nitrogen. The phosphorescence spectra of films were measured in an ARS-4HW cryo equipment (Advanced Research Systems) connecting with a cycle refrigeration of liquid helium.

\subsection{Electrochemical measurements:}

Cyclic voltammetry were performed using a CHI 600E electrochemical analyzer in a gas-tight three-electrode cell at room temperature. A glassy carbon working electrode $(\Phi=5.0 \mathrm{~mm})$, a platinum wire auxiliary electrode, and an $\mathrm{Ag} / \mathrm{Ag}^{+}$reference electrodes $\left(0.1 \mathrm{M} \mathrm{AgNO}_{3}, 0.1 \mathrm{M}\right.$ tetrabutylammonium hexafluorophosphate in acetonitrile) were used. The reduction processes were measured by scanning the potential at a scan rate of $100 \mathrm{mV} / \mathrm{s}$ in acetonitrile, with $0.1 \mathrm{M}$ tetrabutylammonium hexafluorophosphate (TBAP) as supporting electrolyte. The solutions were degassed by purging with $\mathrm{N}_{2}$ gas for approximately 3 minutes before the measurement. The ferrocene couple $\mathrm{Fc}^{+} / \mathrm{Fc}$ was selected as the external reference.

\subsection{Quantum chemical calculations:}

All calculations were performed using the Gaussian 09 program package. ${ }^{4}$ The geometries in the ground state were optimized via DFT calculations at the B3LYP/6-31G* level in vacuum. The reduction state geometries were optimized by DFT/UB3LYP/6-31+G(d). Frequency analysis was used to confirm that the structures are at the local minima of the potential surfaces. At the B3LYP/6-31G(d) level, the LUMO energy level of various bridge groups attached with benzene rings was 
obtained and the natural bond orbitals (NBO) analysis in the ground state geometry was performed. Multiwfn software is used to quantitative analyze the contribution proportions $\left(a_{i}\right)$ of different nonhydrogen atoms to the reduced state SOMO (Table S1). ${ }^{5}$ The reorganization energies $(\lambda)$ were evaluated directly from the adiabatic potential-energy surfaces of neutral/anion species (As shown in Scheme $S 4, \lambda=\lambda_{1}+\lambda_{2}$ ). The single-point calculation on every species was carried out at the B3LYP/6-31+G(d) level. The $\mathrm{T}_{1}$ transition energies of A1-A5, PPT and DPEPO were calculated by TDDFT/B3LYP/6-31G* in dichloromethane based on the ground state geometries, and then corrected by a semi-empirical method reported previously. ${ }^{6}$ The oxidation potentials in dichloromethane and the reduction potentials in acetonitrile were calculated by the thermodynamic cycles method. ${ }^{7}$ For the oxidation and reduction potentials, the exact electron energies were evaluated by PBE0/def2TZVP and M06/6-311++G(d,p) methods, respectively.

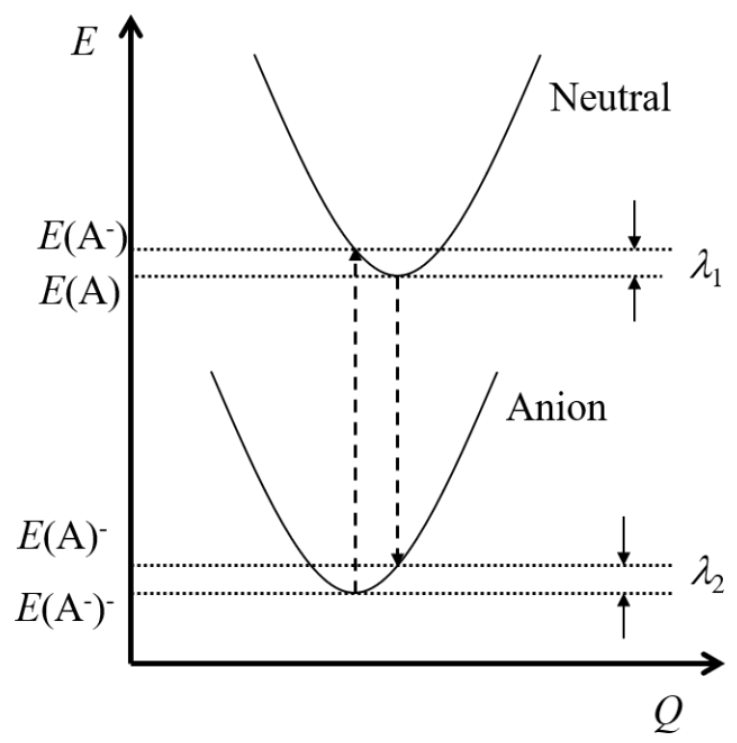

Scheme S4. Schematic description of reorganization energy for electron transfer. The reaction coordinate $Q$ is an ensemble of geometric modifications of the dimer system. 
Table S1. Contribution proportions $\left(a_{i}\right)$ of different nonhydrogen atoms to the reduced state SOMO.

\begin{tabular}{|c|c|c|c|c|c|}
\hline \multicolumn{2}{|c|}{ A1 } & \multicolumn{2}{|c|}{ A2 } & \multicolumn{2}{|c|}{$\mathbf{A 3}$} \\
\hline Atom & $a_{\mathrm{i}}$ & Atom & $a_{\mathrm{i}}$ & Atom & $a_{\mathrm{i}}$ \\
\hline $1(\mathrm{C})$ & 0.05530 & $1(\mathrm{C})$ & 0.07761 & $1(\mathrm{C})$ & 0.06935 \\
\hline $2(\mathrm{C})$ & 0.01855 & $2(\mathrm{C})$ & 0.03230 & $2(\mathrm{C})$ & 0.03748 \\
\hline $3(\mathrm{C})$ & 0.02307 & $3(\mathrm{C})$ & 0.03896 & $3(\mathrm{C})$ & 0.02435 \\
\hline $4(C)$ & 0.02924 & $4(C)$ & 0.04620 & $4(C)$ & 0.03137 \\
\hline $6(C)$ & 0.03060 & $6(C)$ & 0.03105 & $6(C)$ & 0.04261 \\
\hline $10(\mathrm{C})$ & 0.05977 & $10(\mathrm{C})$ & 0.07009 & $10(\mathrm{C})$ & 0.06936 \\
\hline $11(\mathrm{C})$ & 0.01828 & $11(\mathrm{C})$ & 0.04468 & $11(\mathrm{C})$ & 0.02435 \\
\hline $12(\mathrm{C})$ & 0.03044 & $12(\mathrm{C})$ & 0.02606 & $12(\mathrm{C})$ & 0.03748 \\
\hline $13(\mathrm{C})$ & 0.04279 & $13(\mathrm{C})$ & 0.02690 & $13(\mathrm{C})$ & 0.04261 \\
\hline $16(\mathrm{C})$ & 0.05541 & $16(\mathrm{C})$ & 0.06904 & $16(C)$ & 0.06548 \\
\hline $18(\mathrm{C})$ & 0.02734 & $18(\mathrm{C})$ & 0.00569 & $18(\mathrm{C})$ & 0.03137 \\
\hline $19(\mathrm{C})$ & 0.04831 & $19(\mathrm{C})$ & 0.00193 & $19(\mathrm{C})$ & 0.06548 \\
\hline $20(\mathrm{C})$ & 0.04118 & $20(\mathrm{C})$ & 0.00521 & $20(C)$ & 0.00650 \\
\hline $21(\mathrm{C})$ & 0.02892 & $21(\mathrm{C})$ & 0.00332 & $21(\mathrm{C})$ & 0.00228 \\
\hline $22(\mathrm{C})$ & 0.02198 & $23(\mathrm{C})$ & 0.00181 & $22(\mathrm{C})$ & 0.00223 \\
\hline $23(\mathrm{C})$ & 0.01349 & $25(\mathrm{C})$ & 0.00215 & $23(\mathrm{C})$ & 0.00105 \\
\hline $25(\mathrm{C})$ & 0.01925 & $29(\mathrm{C})$ & 0.01374 & $25(\mathrm{C})$ & 0.00080 \\
\hline $27(\mathrm{C})$ & 0.04201 & $30(\mathrm{C})$ & 0.00281 & $27(\mathrm{C})$ & 0.00053 \\
\hline $31(\mathrm{C})$ & 0.00642 & $31(\mathrm{C})$ & 0.00587 & $31(\mathrm{C})$ & 0.02915 \\
\hline $32(\mathrm{C})$ & 0.00215 & $32(\mathrm{C})$ & 0.00364 & $32(\mathrm{C})$ & 0.01570 \\
\hline $33(\mathrm{C})$ & 0.00196 & $34(\mathrm{C})$ & 0.00200 & $33(\mathrm{C})$ & 0.02074 \\
\hline $34(\mathrm{C})$ & 0.00051 & $36(\mathrm{C})$ & 0.00254 & $34(\mathrm{C})$ & 0.01286 \\
\hline $36(\mathrm{C})$ & 0.00065 & $40(\mathrm{C})$ & 0.04991 & $36(C)$ & 0.00886 \\
\hline $38(\mathrm{C})$ & 0.00072 & $41(\mathrm{C})$ & 0.07122 & $38(\mathrm{C})$ & 0.02821 \\
\hline $42(\mathrm{O})$ & 0.00527 & $42(\mathrm{C})$ & 0.00515 & $42(\mathrm{O})$ & 0.00509 \\
\hline $43(\mathrm{P})$ & 0.03832 & $43(\mathrm{C})$ & 0.00256 & $43(\mathrm{P})$ & 0.03839 \\
\hline $45(\mathrm{O})$ & 0.00491 & $44(\mathrm{C})$ & 0.00349 & $44(C)$ & 0.00563 \\
\hline $46(\mathrm{P})$ & 0.04138 & $45(\mathrm{C})$ & 0.00196 & $45(C)$ & 0.01823 \\
\hline $47(C)$ & 0.00808 & $47(\mathrm{C})$ & 0.00105 & $46(C)$ & 0.00564 \\
\hline $48(\mathrm{C})$ & 0.00235 & $49(\mathrm{C})$ & 0.00229 & $47(\mathrm{C})$ & 0.0065 \\
\hline $49(\mathrm{C})$ & 0.00269 & $53(\mathrm{C})$ & 0.00570 & $48(C)$ & 0.00224 \\
\hline $50(\mathrm{C})$ & 0.00113 & $54(\mathrm{C})$ & 0.00340 & $49(\mathrm{C})$ & 0.00228 \\
\hline $52(\mathrm{C})$ & 0.00080 & $55(\mathrm{C})$ & 0.00300 & $50(\mathrm{C})$ & 0.00080 \\
\hline $54(\mathrm{C})$ & 0.00043 & $56(C)$ & 0.00107 & $52(\mathrm{C})$ & 0.00106 \\
\hline $58(\mathrm{C})$ & 0.04317 & $58(\mathrm{C})$ & 0.00179 & $54(\mathrm{C})$ & 0.00053 \\
\hline $59(\mathrm{C})$ & 0.02151 & $60(\mathrm{C})$ & 0.00260 & $58(\mathrm{C})$ & 0.02915 \\
\hline $60(\mathrm{C})$ & 0.03229 & $64(0)$ & 0.01468 & $59(\mathrm{C})$ & 0.01570 \\
\hline $61(\mathrm{C})$ & 0.02181 & $65(\mathrm{P})$ & 0.03788 & $60(C)$ & 0.02074 \\
\hline $63(\mathrm{C})$ & 0.01366 & 66(Si) & 0.05841 & $61(C)$ & 0.01286 \\
\hline
\end{tabular}




$\begin{array}{llllll}65(\mathrm{C}) & 0.04379 & 68(\mathrm{C}) & 0.00686 & 63(\mathrm{C}) & 0.00886 \\ 69(\mathrm{O}) & 0.01689 & 69(\mathrm{C}) & 0.00194 & 65(\mathrm{C}) & 0.02821 \\ & & 70(\mathrm{C}) & 0.00256 & 69(\mathrm{O}) & 0.00509 \\ & 71(\mathrm{C}) & 0.00111 & 70(\mathrm{P}) & 0.03839 \\ & 73(\mathrm{C}) & 0.00096 & 72(\mathrm{~F}) & 0.00159 \\ & 75(\mathrm{C}) & 0.00037 & 73(\mathrm{~F}) & 0.00206 \\ & 79(\mathrm{C}) & 0.02407 & 74(\mathrm{~F}) & 0.00176 \\ & 80(\mathrm{C}) & 0.01253 & 75(\mathrm{~F}) & 0.00176 \\ & 81(\mathrm{C}) & 0.01752 & 76(\mathrm{~F}) & 0.00206 \\ & 82(\mathrm{C}) & 0.01071 & 77(\mathrm{~F}) & 0.00159 \\ & 84(\mathrm{C}) & 0.00698 & & \\ & 86(\mathrm{C}) & 0.02257 & & \\ & 90(\mathrm{O}) & 0.00473 & & \\ & 91(\mathrm{P}) & 0.03687 & & \end{array}$

\begin{tabular}{|c|c|c|c|c|c|}
\hline \multicolumn{2}{|c|}{ A4 } & \multicolumn{2}{|c|}{ A5 } & \multicolumn{2}{|c|}{ DPEPO } \\
\hline Atom & $a_{\mathrm{i}}$ & Atom & $a_{\mathrm{i}}$ & Atom & $a_{\mathrm{i}}$ \\
\hline $1(\mathrm{C})$ & 0.09524 & $1(\mathrm{~S})$ & 0.08085 & $1(\mathrm{C})$ & 0.00080 \\
\hline $2(\mathrm{C})$ & 0.04878 & $2(\mathrm{O})$ & 0.01355 & $2(\mathrm{C})$ & 0.00030 \\
\hline $3(\mathrm{C})$ & 0.05373 & $3(\mathrm{O})$ & 0.01362 & $3(\mathrm{C})$ & 0.00056 \\
\hline $4(C)$ & 0.04963 & $4(C)$ & 0.09627 & $4(\mathrm{C})$ & 0.00031 \\
\hline $6(C)$ & 0.03847 & $5(\mathrm{C})$ & 0.05602 & $6(C)$ & 0.00020 \\
\hline $10(\mathrm{C})$ & 0.01143 & $6(C)$ & 0.05733 & $8(C)$ & 0.00056 \\
\hline $11(\mathrm{C})$ & 0.00413 & $7(\mathrm{C})$ & 0.04449 & $12(\mathrm{C})$ & 0.00105 \\
\hline $12(\mathrm{C})$ & 0.00404 & $9(\mathrm{C})$ & 0.04434 & $13(\mathrm{C})$ & 0.00048 \\
\hline $13(\mathrm{C})$ & 0.00185 & $11(\mathrm{C})$ & 0.09576 & $14(\mathrm{C})$ & 0.00064 \\
\hline $15(\mathrm{C})$ & 0.00166 & $14(\mathrm{C})$ & 0.07933 & $15(\mathrm{C})$ & 0.00021 \\
\hline $19(\mathrm{C})$ & 0.08313 & $15(\mathrm{C})$ & 0.05103 & $17(\mathrm{C})$ & 0.00024 \\
\hline $20(C)$ & 0.04398 & $16(\mathrm{C})$ & 0.04605 & $19(\mathrm{C})$ & 0.00090 \\
\hline $21(\mathrm{C})$ & 0.04664 & $17(\mathrm{C})$ & 0.03447 & $23(\mathrm{C})$ & 0.01756 \\
\hline $22(\mathrm{C})$ & 0.04113 & $19(\mathrm{C})$ & 0.03408 & $24(\mathrm{C})$ & 0.00952 \\
\hline $24(\mathrm{C})$ & 0.03213 & $21(\mathrm{C})$ & 0.07723 & $25(\mathrm{C})$ & 0.01543 \\
\hline $28(\mathrm{O})$ & 0.01140 & $24(\mathrm{C})$ & 0.00421 & $26(C)$ & 0.01101 \\
\hline $29(\mathrm{P})$ & 0.07234 & $25(\mathrm{C})$ & 0.00133 & $27(\mathrm{C})$ & 0.00753 \\
\hline $30(\mathrm{C})$ & 0.09067 & $26(C)$ & 0.00244 & $29(\mathrm{C})$ & 0.02302 \\
\hline $31(\mathrm{C})$ & 0.07805 & $27(\mathrm{C})$ & 0.00148 & $33(\mathrm{O})$ & 0.00210 \\
\hline $32(\mathrm{C})$ & 0.00314 & $29(\mathrm{C})$ & 0.00083 & $34(\mathrm{P})$ & 0.00663 \\
\hline $33(\mathrm{C})$ & 0.00262 & $31(\mathrm{C})$ & 0.00178 & $35(\mathrm{C})$ & 0.06724 \\
\hline $34(\mathrm{C})$ & 0.00162 & $35(\mathrm{C})$ & 0.00684 & $36(C)$ & 0.04328 \\
\hline $35(\mathrm{C})$ & 0.00088 & $36(\mathrm{C})$ & 0.00241 & $37(\mathrm{C})$ & 0.11630 \\
\hline $36(C)$ & 0.00034 & $37(\mathrm{C})$ & 0.00145 & $38(\mathrm{C})$ & 0.12416 \\
\hline $38(\mathrm{C})$ & 0.00035 & $38(\mathrm{C})$ & 0.00095 & $40(C)$ & 0.08119 \\
\hline
\end{tabular}




$\begin{array}{rlllll}40(\mathrm{C}) & 0.00010 & 40(\mathrm{C}) & 0.00088 & 41(\mathrm{C}) & 0.03829 \\ 44(\mathrm{C}) & 0.00268 & 42(\mathrm{C}) & 0.00069 & 45(\mathrm{C}) & 0.01223 \\ 45(\mathrm{C}) & 0.00091 & 46(\mathrm{O}) & 0.00360 & 46(\mathrm{C}) & 0.01034 \\ 46(\mathrm{C}) & 0.00172 & 47(\mathrm{P}) & 0.02580 & 47(\mathrm{C}) & 0.00464 \\ 47(\mathrm{C}) & 0.00030 & 48(\mathrm{C}) & 0.00334 & 48(\mathrm{C}) & 0.00341 \\ 49(\mathrm{C}) & 0.00040 & 49(\mathrm{C}) & 0.00118 & 50(\mathrm{C}) & 0.00758 \\ 51(\mathrm{C}) & 0.00090 & 50(\mathrm{C}) & 0.00193 & 52(\mathrm{C}) & 0.00603 \\ 55(\mathrm{C}) & 0.00338 & 51(\mathrm{C}) & 0.00036 & 56(\mathrm{C}) & 0.05741 \\ 56(\mathrm{C}) & 0.00199 & 53(\mathrm{C}) & 0.00044 & 57(\mathrm{C}) & 0.04036 \\ 57(\mathrm{C}) & 0.00123 & 55(\mathrm{C}) & 0.00013 & 58(\mathrm{C}) & 0.03010 \\ 58(\mathrm{C}) & 0.00044 & 59(\mathrm{C}) & 0.00377 & 59(\mathrm{C}) & 0.01534 \\ 60(\mathrm{C}) & 0.00033 & 60(\mathrm{C}) & 0.00138 & 61(\mathrm{C}) & 0.02281 \\ 62(\mathrm{C}) & 0.00018 & 61(\mathrm{C}) & 0.00212 & 63(\mathrm{C}) & 0.05244 \\ 66(\mathrm{C}) & 0.00373 & 62(\mathrm{C}) & 0.00047 & 67(\mathrm{O}) & 0.01346 \\ 67(\mathrm{C}) & 0.00163 & 64(\mathrm{C}) & 0.00053 & 68(\mathrm{P}) & 0.08180 \\ 68(\mathrm{C}) & 0.00219 & 66(\mathrm{C}) & 0.00019 & 69(\mathrm{O}) & 0.01039 \\ 69(\mathrm{C}) & 0.00056 & 70(\mathrm{O}) & 0.01464 & & \\ 71(\mathrm{C}) & 0.00078 & 71(\mathrm{P}) & 0.03855 & & \\ 73(\mathrm{C}) & 0.00038 & & & & \\ 77(\mathrm{C}) & 0.00056 & & & & \\ 78(\mathrm{C}) & 0.00022 & & & & \\ 79(\mathrm{C}) & 0.00026 & & & & \\ 80(\mathrm{C}) & 0.00011 & & & & \\ 82(\mathrm{C}) & 0.00013 & & & & \\ 84(\mathrm{C}) & 0.00027 & & & & \\ 88(\mathrm{C}) & 0.00019 & & & & \\ 89(\mathrm{C}) & 0.00026 & & & & \\ 90(\mathrm{C}) & 0.00015 & & & & \\ 91(\mathrm{C}) & 0.00020 & & & & \\ 93(\mathrm{C}) & 0.00026 & & & & \\ 95(\mathrm{C}) & 0.00090 & & & & \\ 99(\mathrm{O}) & 0.00020 & & & & \\ 100(\mathrm{O}) & 0.01185 & & & & \\ 101(\mathrm{O}) & 0.01493 & & & & \\ 102(\mathrm{P}) & 0.00138 & & & & \\ 103(\mathrm{P}) & 0.03835 & & & & \\ 104(\mathrm{P}) & 0.03055 & & & & \\ \end{array}$

\subsection{Device fabrication and measurements:}

After the pre-cleaned indium tin oxide (ITO) glass substrates had been treated with ultraviolet-ozone for 15 minutes, the inorganic, organic and metal layers were 
thermally evaporated onto the substrates in an inert chamber under a pressure of $2 \times 10^{-4} \mathrm{~Pa}$. The deposition rates were $0.1 \AA$ /s for $\mathrm{MoO}_{3}$ and Liq layer, 1-2 $\AA$ /s for organic layers, and $4 \AA$ /s for Al layer. The current density, voltage and luminance characteristics of the devices were measured in ambient air with a Keithey 2400 Source meter and a Keithey 2000 Source multimeter equipped with a calibrated silicon photodiode. The electroluminescence spectra were recorded using a multichannel spectrometer (PMA12, Hamamatsu Photonics). Assuming Lambertian emission, the external quantum efficiency can be calculated from the luminance, current density, and EL spectrum.

\section{References}

(1) Wang, Z. B.; Helander, M. G.; Greiner, M. T.; Qiu, J.; Lu, Z. H. Carrier mobility of organic semiconductors based on current-voltage characteristics. J. Appl. Phys. 2010, 107, 034506.

(2) Fawcett, J.; Platt, A. W. G.; Vickers, S.; Ward, M. D. Lanthanide halide and nitrate complexes with potentially tridentate bisphosphine dioxide-ether donors. Polyhedron 2004, 23, 2561-2567.

(3) Zhang, J.; Ding, D.; Wei, Y.; Xu, H. Extremely condensing triplet states of DPEPO-type hosts through constitutional isomerization for high-efficiency deep-blue thermally activated delayed fluorescence diodes. Chem. Sci. 2016, 7, 2870-2882.

(4) Frisch, M. J.; Trucks, G. W.; Schlegel, H. B.; Scuseria, G. E.; Robb, M. A.; Cheeseman, J. R.; Scalmani, G.; Barone, V.; Mennucci, B.; Petersson, G. A.; Nakatsuji, H.; Caricato, M.; Li, X.; Hratchian, H. P.; Izmaylov, A. F.; Bloino, J.; Zheng, G.; Sonnenberg, J. L.; Hada, M.; Ehara, M.; Toyota, K.; Fukuda, R.; Hasegawa, J.; Ishida, M.; Nakajima, T.; Honda, Y.; Kitao, O.; Nakai, H.; Vreven, T.; Montgomery, Jr., J. A.; Peralta, J. E.; Ogliaro, F.; Bearpark, M.; Heyd, J. J.; Brothers, E.; Kudin, K. N.; Staroverov, V. N.; Keith, T.; Kobayashi, R.; Normand, J.; Raghavachari, K.; Rendell, A.; Burant, J. C.; Iyengar, S. S.; Tomasi, J.; Cossi, M.; Rega, N.; Millam, J. M.; Klene, M.; Knox, J. E.; Cross, J. B.; Bakken, V.; Adamo, C.; 
Jaramillo, J.; Gomperts, R.; Stratmann, R. E.; Yazyev, O.; Austin, A. J.; Cammi, R.; Pomelli, C.; Ochterski, J. W.; Martin, R. L.; Morokuma, K.; Zakrzewski, V. G.; Voth, G. A.; Salvador, P.; Dannenberg, J. J.; Dapprich, S.; Daniels, A. D.; Farkas, O.; Foresman, J. B.; Ortiz, J. V.; Cioslowski, J.; Fox, D. J. Gaussion 09, Revision D.01; Gaussian, Inc.: 2009.

(5) Lu, T.; Chen, F. Multiwfn: A multifunctional wavefunction analyzer. J. Comput. Chem. 2012, 33, 580-592.

(6) Huang, S.; Zhang, Q.; Shiota, Y.; Nakagawa, T.; Kuwabara, K.; Yoshizawa, K.; Adachi, C. Computational Prediction for Singlet- and Triplet-Transition Energies of Charge-Transfer Compounds. J. Chem. Theory Comput. 2013, 9, 3872-3877.

(7) Wang, D.; Huang, S.; Wang, C.; Yue Y.; Zhang, Q. Computational prediction for oxidation and reduction potentials of organic molecules used in organic light-emitting diodes. Org. Electron. 2019, 64, 216-222. 